\title{
The role of architectural design for rheumatic patients' wellbeing: the point of view of Environmental Psychology
}

\author{
L. Punzi1 ${ }^{1,2}$, M. Chia ${ }^{1}$, S. Cipolletta ${ }^{3}$, C. Dolcetti ${ }^{1}$, P. Galozzi ${ }^{1,4}$, O. Giovinazzi ${ }^{5}$, \\ S. Tonolo ${ }^{6}$, R. Zava ${ }^{3}$, F. Pazzaglia ${ }^{1,3}$ \\ ${ }^{1}$ Health and Motion Venice Association (HEMOVE Onlus), Venice, Italy; ${ }^{2}$ Center for Gout and Metabolic Bone \\ and Joint Diseases, Rheumatology, SS Giovanni and Paolo Hospital, Venice, Italy; ${ }^{3}$ Department of General \\ Psychology, University of Padova, Padova, Italy; ${ }^{4}$ Department of Medicine DIMED, University of Padova, \\ Padova, Italy; ${ }^{5}$ Architect, Ph.D. in Urban Planning and Public Policies for the Territory, IUAV University of \\ Venice; ${ }^{6}$ Associazione Nazionale Malati Reumatici (ANMAR; National Association of Rheumatic Patients), Italy
}

\section{SUMMARY}

Rheumatic diseases (RD) are among the most frequent disorders in the population and the major causes of chronic pain and disability. The resulting consequences are catastrophic, leading to a significant socio-economic burden, which includes significant reductions in quality of life (QoL) and limitations in regular work and daily activities of patients. In spite of this, rheumatic diseases are often misunderstood or diagnosed late, probably due to their characteristics of silent diseases, sometimes unrecognizable to unaffected or unskilled people. Actually, it is surprising that, despite their consequences on QoL and on individual impact, rheumatic diseases are underestimated by the public opinion, which is probably more attracted by other major diseases causing death. This silent perception can even be seen in some among the most recent psycho-social approaches to population needs in the fields of Health Psychology and Environmental Psychology. The latter, also known as Architectural Psychology, is a branch of Psychology that analyses the effects of the built environment on humans, including those affected by diseases. Paradoxically, in many cases, some components of the environments created to protect individuals and/or the population may represent barriers and subsequently causes of disability and suffering in patients with rheumatic diseases. In order to increase awareness about this particular aspect of social life, HEMOVE Onlus, a non-profit association, has promoted the creation of a multidisciplinary Task Group, which included mainly rheumatologists, psychologists and architects, with the aim of applying also for the benefit of rheumatic patients the most modern technical skills available in the context of Environmental Psychology, including in particular design and information technology.

Key words: Rheumatology; rheumatic diseases; arthritis; pain; psychology; environmental psychology; architectural psychology.

Reumatismo, 2020; 72 (1): 60-66

\section{INTRODUCTION}

heumatic diseases (RD) are the more frequent disorders in the population and the major causes of chronic pain and disabilities (1-3). In the US, the Center for Disease Control and Prevention estimated that 54.4 million adults are affected by arthritis, approximately $25 \%$ of the population. In Italy, $27 \%$ of people aged 18 or older claim past or current occurrence of musculoskeletal pain for at least a month (4). It is estimated that disabilities due to RD increased by $45 \%$ from 1990 to 2010 , mainly due to arthritis and low back pain.
This rate is expected to grow further in the coming years due to an increase in obesity, physical inactivity and aging (5). The consequences may be catastrophic and lead to a significant socio-economic burden, which includes significant reductions in quality of life (QoL) and limitations in the regular work and daily activities (6). In spite of this, RDs are often misunderstood or diagnosed late, probably due to their characteristics of silent diseases. Although patients with RDs report pain and disability, they give the impression to be healthy. However, RDs have significant repercussions on a very large number of subjects - the 
patients themselves, their family members, the healthcare professionals and providers, etc. - and have a significant impact on their lives. In a report on musculoskeletal diseases, the World Health Organization (WHO) concluded that although fatal diseases attract much more public attention, $R D s$ or musculoskeletal disorders are the most frequent in the world population, with undoubted repercussions on health and quality of life, causing a huge load of costs for health care services (7). Only recently has the social impact of RDs been taken into consideration. The role of the environment and in particular of architecture is still neglected in relation to RDs, despite their undoubted role in society and for the improvement of QoL. Furthermore, architecture can facilitate or limit the access of RD patients to two of the most important factors in life: beauty and movement.

\section{Unmet needs for rheumatic patients. Quality of life and silent disability}

The WHO defines QoL as a rather broad concept characterized by complete physical, mental and social well-being, associated with a good level of independence, social relations and the ability to relate to the environment, and not merely as the absence of disease or infirmity (8). Patients with RDs report reduced QoL in many areas, including physical health, psychological status, level of independence, social relationships and interaction with the environment, and personal beliefs or perceptions, compared to the normal population (9). A recent study has shown that patients with rheumatoid arthritis (RA) essentially perceived the concept of QoL in three different ways, i.e. in terms of independence, emancipation and participation (10). These categories can in turn be described as physical, psychological and social, and fall within the WHO definition of QoL.

In the category of the independent patients, the focus is on the physical aspects that provide independence in terms of physical function and economic consequences. In the category of the emancipated patients, the core is the different psychological aspects involved in the management of per- sonal life, in particular the ability to cope in everyday life. In the category of the participant patients, the concepts are focused on various social aspects, such as participation and belonging. The participant patient describes the concepts of loneliness and fear of being excluded and not helped. These categories can be applied to other RDs and demonstrate the close relationship between the RD and the environment with which patients constantly interact and to which they must adapt.

Patients with disabilities, including those with RDs, can generally be divided into two main groups, i.e. patients with or without legally-recognized disabilities (11). A disability can be legally confirmed by an authority that is entitled to establish the degree of disability. A biological disability is instead a subjective state in which the person feels to be limited in carrying out basic activities without having a disability certificate. Not only do RDs influence the ability to work, but also the daily life of these subjects and therefore their independence. The most common problems reported by patients with RD include getting dressed, getting up, turning on a tap, opening a bottle cap, getting on the bus and not having a place to sit, difficulty in climbing the stairs and opening heavy doors, and inability to stand for a long period of time (11). All these aspects are often underestimated not only by the authorities, but also by the clinicians, e.g. when setting an appointment for outpatient visits.

Almost all daily activities of patients with rare diseases are related to the environment that can be influenced in turn by their socioeconomic status (SES). This is associated with worse clinical progression, decreased functional capacity and reduced QoL (12, 13). The SES has been defined as a rather broad concept that refers to people, their families and groups of population with respect to their ability to create or consume goods that are valuable in our society (13). This type of disability is frequently overlooked in architecture schools, with obvious consequences on the design of buildings and common objects. Severe disability in RDs, not silent disability, may progress 
significantly. A lot of studies on the value of physical and motor activities and home exercises in patients with arthritis rarely take into account the role of architectural barriers, especially in small houses, which reduce their consistency in performing these exercises.

Women with RA need assistance from relatives or friends much more often than men (women 65\%, men 25\%). They require help for various situations, such as housework (70\%), shopping (41\%), lifting heavy objects $(20 \%)$, transportation $(15 \%)$, opening jars or bottles $(15 \%)$ and personal hygiene $(11 \%)$. Furthermore, patients are often financially dependent on other people (family, friends) $(10,12)$.

\section{An interdisciplinary approach to environment, health and well-being} Taking care of places where people live, work or spend their leisure time practically means to be able to support them throughout their life. This can give a significant contribution to the improvement of the quality of life of individuals affected by chronic diseases, such as RDs. However, in order to achieve this goal, it is necessary to develop an interdisciplinary approach that relies on evidence from different, yet complementary disciplines, such as medicine, environmental and health psychology, architecture, design, engineering and information technology, and lead to appropriate solutions.

However, in order to develop this approach, it is necessary to take into account some challenges that contemporary society is facing: public health, chronic disorders, environmental quality, space planning, social inequality, among others, all strongly correlated to global demographic phenomena such as the increase in the urban population and the accelerated aging of the population. In the coming years public health and the quality of the environment may be influenced also by living conditions in cities, public and private spaces, political choices, urban planning approaches, architectural design criteria, regulatory and structural interventions, and promotion of lifestyles intended to protect the health of citizens and the quality of the environment in which they live.

\section{Environmental psychology: history and topics}

Among the disciplines mentioned above, the lesser-known and with an unexplored potential is Environmental Psychology (EP), a branch of Psychology that started to develop in the 1960s. Initially, it was referred to as Architectural Psychology due to the strong focus on the effects of the built environment on humans. Given the increased numbers of topics under study, the more general term Environmental Psychology was preferred. The two terms are currently used as synonyms. The EP studies the interaction between human beings and their environments, analyzing both the environmental impact of human behaviors and the effects that natural and built environments have on emotions, behaviors, lifestyles and wellness (14).

The physical characteristics of the environment can influence positively, but also negatively the autonomy, the behavior, the emotions and the well-being of rheumatic patients. Hence, it is important to investigate the interactions between a trusted environment and the rheumatic diseases from an interdisciplinary perspective, with contributions from various disciplines such as medicine, psychology and architecture.

\section{The interaction between \\ the individuals and their environment: the docility hypothesis}

The ecological model of interaction between the individual and the environment considers the process of adaptation of the individual to his environment as deriving from the interaction between the environmental competence and the environmental pressure. The former depends on the set of individual sensory, motor and cognitive resources, the latter corresponds to the set of environmental characteristics that influence the adaptive process of the individual to his environment (15). A good adaptation is a guarantee of well-being, autonomy, quality of life, and it is maximum, when competence and environmental pressure 
are in perfect balance (level of adaptation). In the presence of a vulnerable condition, as in a chronic disease, it can diminish competences and, simultaneously, increase the burden of environmental pressure. The consequence is greater dependence on the environment (docility hypothesis) (15).

For these reasons, it is important that the physical and social environment can satisfy the needs of many people, including those with sensory, physical and cognitive disabilities, in the perspective that an inclusive environment would be a better environment not only for people with disabilities but for everyone.

\section{Universal design: a project for everyone}

In many cases, the context in itself may become a disabling factor. An urban place, a living space or an object can deprive the users of the possibility or ability to perform a task and to achieve their own goal. Sometimes, people with disabilities and in particular those affected by RDs are exposed to environment or common objects that can limit their actions. Greater attention to the demand for integration from people with disabilities is a cornerstone of the culture based on Universal Design (UD), which has become a reference in the design of environmental and built spaces, in the creation of new products, in research and training, as well as in the field of communication. The culture of UD started at the Center for Universal Design (CUD) of the North
Carolina State University (USA), and was developed in 1997 by a group of experts (architects, designers, engineers, environmental planners and researchers) under the guidance of Ronald Mace. This approach promotes an accessible and universal project oriented towards inclusiveness, in which the differences between users are considered expressions of an individual specialization. In this perspective, the project does not lead to the "sectorization" of dedicated, exclusive or specialized areas, but it favors the usefulness of the space in an undifferentiated manner for the benefit of everyone.

UD is hinged on seven principles (16), which are aimed to achieve high standards for all users, but also greater sensitivity in the design process for weak users. (Table I) This approach requires multidisciplinary skills, coordinated actions and social responsibility so as to use new knowledge and the most advanced technologies to ensure compliance to codes and standards. It also requires the designer to know, understand and interpret - not only with the contribution of other experts, but also of users themselves - the skills, needs, preferences, individual characteristics to be valued, etc. The ambition of UD is to go beyond the application of rules and standards stimulating innovative and creative solutions in order to promote strategies and choices that meet the expectations of the largest number of users, to guarantee that the pro-

Table I - Universal design principles.

\begin{tabular}{|l|l|}
\hline PRINCIPLE ONE: Equitable Use & The design is useful and marketable to people with diverse abilities. \\
\hline $\begin{array}{l}\text { PRINCIPLE TWO: } \\
\text { Flexibility in Use }\end{array}$ & $\begin{array}{l}\text { The design accommodates a wide range of individual preferences and } \\
\text { abilities. }\end{array}$ \\
\hline $\begin{array}{l}\text { PRINCIPLE THREE: } \\
\text { Simple and Intuitive Use }\end{array}$ & $\begin{array}{l}\text { Use of the design is easy to understand, regardless of the user's } \\
\text { experience, knowledge, language skills, or current concentration level. }\end{array}$ \\
\hline $\begin{array}{l}\text { PRINCIPLE FOUR: } \\
\text { Perceptible Information }\end{array}$ & $\begin{array}{l}\text { The design communicates necessary information effectively to the user, } \\
\text { regardless of ambient conditions or the user's sensory abilities. }\end{array}$ \\
\hline $\begin{array}{l}\text { PRINCIPLE FIVE: } \\
\text { Tolerance for Error }\end{array}$ & $\begin{array}{l}\text { The design minimizes hazards and adverse consequences of accidental } \\
\text { or unintended actions. }\end{array}$ \\
\hline $\begin{array}{l}\text { PRINCIPLE SIX: } \\
\text { Low Physical Effort }\end{array}$ & $\begin{array}{l}\text { The design can be used efficiently and comfortably and with minimum } \\
\text { effort. }\end{array}$ \\
\hline $\begin{array}{l}\text { PRINCIPLE SEVEN: Size } \\
\text { and Space for Approach and Use }\end{array}$ & $\begin{array}{l}\text { Appropriate size and space are provided for approach, reach, } \\
\text { manipulation, and use regardless of user's body size, posture, or mobility. }\end{array}$ \\
\hline
\end{tabular}

Copyright 1997 NC State University, The Center for Universal Design. 
ject runs smoothly without necessarily affecting aesthetic aspects, and to promote a change in of perspective, also in quality of life. This can offer everyone opportunities for access and use on the basis of one's personal inclinations. These design principles can be transformed into a concrete response to many environmental problems, especially for all those who are unable to interact freely with the surrounding environment due to their illness. This approach can allow many people to cope better with their everyday life.

Among the many interesting projects in the field of architecture, we can mention those developed by the Isala Klinieken Hospital in Zwolle (Netherlands) or the Children's Hospital of Pittsburgh in Pennsylvania (US), which are focused on both physical and emotional aspects. Their aim was to start from the direct experience with the phenomena and from the analysis of the needs of potential users, who are involved all along the process. In these projects, also the role of the associations is fundamental. A different experience in the field of engineering is the FabLab, introduced a decade ago at the MIT's Media Lab. FabLab platforms are well-suited to support interdisciplinary, multifaceted and integrated skill development, implementing scientific knowledge, while gaining relevant technical skills and developing the art of creative thinking (17). By using accessible and relevant technology for design, engineering and prototyping, the labs provide a platform which can involve both conceptual thinkers and practical doers. FabLab platforms are becoming also a new way of increasing opportunities to find proper solutions for patient needs by involving all the stakeholders even from the project building phase.

\section{User-centered design culture:} a virtuous approach to rheumatic diseases and role of patients' associations Both in design and architectural psychology, some projects have emerged in recent years aimed to improve the quality of life of patients with RDs. Among these, the initiative of Politecnico di Milano with $\mathrm{No}$
Non Ci Fermiamo, a +ABILITY project in collaboration with some associations, like ANMAR (National Association of Rheumatic Diseases), ALOMAR (Lombard Association for Rheumatic Patients) and APMAR (Association for the Rheumatic Rare Diseases), that aim to shape and create tools, aids or, even better, design products dedicated to a specific category of people, although they can be used by everyone, exploiting the principles of the UD. This work is based in particular on a co-design activity between patients, designers, doctors and therapists, necessary to redesign those objects perceived by the patient as not very functional. As defined by Marinella Levi, professor at the Milan Polytechnic and project leader, the idea is to start with a design concept for everyone and evolve up to a design concept with everyone. Starting from ideas of all people involved in the project, objects useful to all users with or without disabilities are created. The conceived products are then made using 3D printers. To improve these objects, more information is collected with questionnaire available online (http://piulab.it/).

In a recently published study on the architectural context, Valeria Tatano, professor at IUAV, deals with the issue of inclusive accessibility within the city of Venice from the point of view of all people who live there and face a series of obstacles every day (18). Among several initiatives held in Venice, in recent years, one envisaged the collection of suggestions from some RD patients through a questionnaire on three facilitated access ramps built for the Venice Marathon (19). The RD patients expressed their satisfaction with this initiative aimed at facilitating their accessibility, yet asked for some improvements, such as reducing slippery areas and increasing the usability of catwalks. Many improvements have been or will be made, even with the fundamental contribution of various patients' associations. Patient organizations should also be given the role of mediators between the multidisciplinary stakeholders in scientific publications. Including the perspective of patients in scientific works is the first step to fill the gap, which can be found in 
healthcare literature between the perspective of healthcare providers and that of patients. This is becoming very important also at the international level in the European rare disease networks (ERN) (20).

This user-centered design culture can be taken as reference to understand the importance of a multidisciplinary approach in the creation of private and/or public spaces or objects that can be usable and accessible to as many users as possible. The non-profit association HEMOVE (Health and Motion Venice Organization) is trying to act as a link between the users so as to facilitate collaboration between current and potential patients and the different disciplines involved in the conception and construction of these architectural tools from the point of view of prevention, using the latest insights from architectural psychology studies.

\section{CONCLUSIONS}

Rheumatic diseases are among the most common causes of chronic disability and are expected to increase further with the aging of the population, therefore highlighting the need to limit the growing number of people suffering from pain and disability. Changes in lifestyle, especially in terms of QoL, can influence the course of some RDs, thus preventing and improving the disease progression. In turn, the lifestyle depends on the type of environment, which involves architectural aspects and the socio-economic status that can interact with each other. Although important, many of these aspects have so far been neglected, leading to some significant disparities, even in many apparently egalitarian societies, and resulting into inequalities even in terms of access to health care and type of disease progression. Therefore, not only is the access to appropriate prevention and treatments for chronic diseases, including RDs, necessary, but it is also one of the basic rights of individuals. Consequently, the influence of architecture as a major determinant of well-being for RD patients deserves to be carefully considered in future studies and proposed to our authorities and/ or those involved in the treatment of rare diseases.

\section{Contributions}

LP, MC, RZ and FP conceived and designed the study. SC, CD, PG, OG, and ST wrote the initial manuscript. All authors have reviewed and approved the final manuscript.

\section{Conflict of interests}

The authors declare no potential conflict of interests.

\section{Funding}

The study was supported by HEMOVE (Health and Motion Venice Association) Onlus, a non-profit association involved in the scientific dissemination and prevention of disability associated with rheumatic diseases.

\section{REFERENCES}

1. Woolf AD, Erwin J, March L. The need to address the burden of musculoskeletal conditions. Best Pract Res Clin Rheumatol. 2012; 26: 183-224.

2. Hoy DG, Smith E, Cross M, et al. The global burden of musculoskeletal conditions for 2010: an overview of methods. Ann Rheum Dis. 2014; 73: 982-9.

3. Mont S, Caporali R. Chronic pain: the burden of disease and treatment innovation. Reumatismo. 2015; 67: 35-44.

4. Cimmino MA, Parisi M, Moggiana GL, et al. Prevalence of self-reported peripheral joint pain and swelling in an Italian population: The Chiavari study. Clin Exp Rheumatol. 2001; 19: 35-40.

5. Vos T, Flaxman AD, Naghavi M, et al. Years lived with disability (YLDs) for 1160 sequelae of 289 diseases and injuries 1990-2010: a systematic analysis for the Global Burden of Disease Study 2010. Lancet. 2012; 380: 2163-96.

6. Branco JC, Rodrigues AM, Gouveia N, et al. Prevalence of rheumatic and musculoskeletal diseases and their impact on health-related quality of life, physical function and mental health in Portugal: results from EpiReumaPt - a national health survey. RMD Open. 2016; 2: e000166.

7. The WHO Scientific Group. The burden of muskuloskeletal conditions at the start of the new millenium. WHO Technical Report Series 919, WHO, Geneva, 2003.

8. The WHOQOL Group. The World Health Organization Quality of Life assessment 
(WHOQOL): position paper from the World Health Organization. Soc Sci Med. 1995; 41: 1403-9.

9. Kvien TK, Uhlig T. Quality of life in rheumatoid arthritis. Scand J Rheumatol. 2005; 34: 333-41.

10. Malm K, Bergman S, Andersson MLE, et al. Quality of life in patients with established rheumatoid arthritis: A phenomenographic study. SAGE Open Med. 2017; 5: 1-8.

11. Kłak A, Raciborski F, Samel-Kowalik P. Social implications of rheumatic diseases. Reumatologia. 2016; 54: 73-8.

12. Lapsley HM, March LM, Tribe KL, et al. Living with rheumatoid arthritis: expenditures, health status, and social impact on patients. Ann Rheum Dis. 2002; 61: 818-21.

13. Suzanne M, Verstappen M. The impact of socio-economic status in rheumatoid arthritis. Rheumatology. 2017; 56: 1051-2.

14. Steg L, de Groot J. Environmental psychology: An introduction ( $2^{\text {nd }}$ Ed.). Hoboken, New Jersey, Stati Uniti: Wiley; 2019.
15. Lawton MP, Nahemow L. Ecology and the aging process. In: Eisdorfer C, Lawton MP, eds. The psychology of Adult Development and Aging, Washington (D.C.): American Psychology Association. 1973; 619-74.

16. The Center for Universal Design. The Principles of Universal Design, Version 2.0. Raleigh, NC: North Carolina State University. 1997.

17. Stephenson MK, Dow DE. The community FabLab platform: applications and implications in biomedical engineering. Conf Proc IEEE Eng Med Biol Soc. 2014; 2014: 1821-5.

18. Tatano V. Atlante dell'accessibilità urbana a Venezia. Conegliano, Italia: Anteferma Edizioni; 2019.

19. Zava R. L'accessibilità nelle città storiche: uno studio su Venezia. Master Degree Diss., Università di Padova, Italy; 2017.

20. Limper M, Scirè CA, Talarico R, et al. Antiphospholipid syndrome: state of the art on clinical practice guidelines. RMD Open. 2018; 4: e000785. 